the grading of the several branches of the curriculum, For example, the first year of clinical instruction. which would correspond with the second year of medical study, should aim to make the student personally familiar with the physiogonomy and symptomatology of diseases, and with the use of all instruments and appliances for accuracy of examination and diagnosis, both medical and surgical. The second clinical year should be chiefly occupied in the study of the actual pathological processes taking place in the different forms of disease and their relation to the symptoms, including personal examinations, chemical and microscopic, of the secretions and excretions in the several stages of progress of disease, as well as the tissue changes to be found after death. With these studies, the objects to be accomplished for counteracting the morbid processes, correcting the secretions, and preventing permanent or fatal tissue changes, should be clearly indicated. Having thus become clinically familiar with the symptoms, diagnosis and pathology of diseases and the indications desirable to fulfill in their management, the third clinical year should be devoted directly to the study of methods and means of treatment, preventive, hygienic, therapeutic and surgical.

The advantages to be gained by such grading of clinical studies as I have just indicated must be obvious to both the student and the clinical teacher: 1. By giving to each year of clinical study, limited and definite objects, the accomplishment of which makes his next year's work easier and more thorough, you not only make the student's practical knowledge more systematic and comprehensive, but you add much to his mental discipline and accuracy of observation. 2. Such a system necessarily limits the number of students in any one clinical class, and in the same ratio increases the opportunities and value of individual training, which is the most essential feature of all true clinical study. The dispensaries and hospitals of our larger cities in which the medical schools are chiefly located, contain an abundance of clinical material, that needs only judicious arrangement and faithful attention to secure both the most complete clinical instruction and the most skilful service for the sick. But as the subject is to be before you for further discussion, I will not occupy your time with details at present. The principle or system of grading the clinical instruction of students during their consecutive courses of medical college attendance, will be found no less important and advantageous than is the proper grading of the various branches of medicine contained in the college curriculum.

\section{THE KIND AND AMOUNT OF LABORATORY WORK WHICH SHOULD BE REQUIRED IN OUR MEDICAL SCHOOIS.}

BY VICTOR C. VAUGHAN, A.M., M.D., DEAN MEDICAL DEPARTMENT UNIVERSITY OF MICHIGAN.

$M r$. President and Gentlemen:-It will not be necessary for me to take any time in giving the arguments in favor of teaching medical students by the laboratory method many of the sciences which must enter into the curriculum of study. I will take it for granted that all reputable teachers of medicine understand that analytical chemistry, practical anatomy, bacteriology, histology, physiology, analysis of urine, pathology, etc., must be taught in the laboratory in part at least, if taught at all. How can you expect a practitioner to tell whether a given bit of tissue is from a malignant growth or not, when he has never seen a cancer cell? How could he be trusted with such an examination when he has never used a microscope, cut a section, or made a microscopical mount? How can the young practitioner diagnositicate tuberculosis in its early stages, when he has had no practical experience in staining germs and when he would not recognize the bacillus tuberculosis should it be placed before him? How can he detect tyrosine or leucine in the urine, when he does not know a crystal from an air-bubble? How can he recognize structural disease of the kidney when he does not know a cast from an epithelial cell? These questions suggest their own answers. It might be said, possibly, that many of the older men now practicing medicine and doing so satisfactorily, and, indeed, with credit to themselves and the profession, never had laboratory instruction in these branches. This is true, but these men have been compelled to take up these new studies and methods for themselves. Besides, more is expected in these directions from the recent graduate than from the older man. Medical schools should always give the best and the most recent information, and every fact which may possibly aid in the diagnosis or treatment of disease should be made known to the student. The student has the right to expect and to demand this of teachers, and all intelligent and conscientious students will do so. The time once was when the teacher was a model from which his students copied. The student measured his own success by the extent to which he imitated his master. Now, that teacher who does not give his student opportunities for independent and original work in science is to a large extent a failure. Just as in the general progress of the race, one generation should be wiser than the preceding, so every class should contain one or more students who will at maturity be wiser than the best teacher. I will presume that all of us here agree that laboratory instruction should be given in medical schools, and with this presumption I will now turn to the question of what, in kind and amount, should this laboratory teaching consist.

We will suppose that the student has had a full course in a good high school or an equivalent of this. (The best medical colleges are not satisfied with a less requirement for admission.) The student is able to use the English language correctly, and he has a good drill in mathematics, including arithmetic, algebra and plain geometry. He has had, we will suppose, fair instruction in systematic botany zoology. He is acquainted with the general classification of plants and animals, and knows the meaning of the general terms employed in the natural sciences. He has some knowledge of physics, knows what is meant by the conservation of energy, and understands the fundamental principles underlying our knowledge of heat, light and electricity. I am aware of the fact that many medical schools admit students who have not these qualifications, but such a school does not comply with the rules of this Association, and I am to discuss the question of laboratory teaching in those colleges which belong to this Association and which live up to the requirements to which we have mutually and voluntarily pledged ourselves. 
In the first place it will be convenient to specify what I mean when I speak of the number of hours, days or weeks given to a subject. We will suppose that there are five teaching days in the week, that the forenoon of these days are devoted to class instruction given in the form of lectures and recitations, and that four hours of each afternoon are spent in laboratory work. In certain schools this order may be reversed or some of the laboratory instructions, practical anatomy, for instance, may be given in the evening. With the understanding then that four hours shall constitute a day of laboratory work, I will outline such a course as I conceive to be essen. tial to the student.

The courses of laboratory work which should be embraced in the curriculum are, 1. Analytical chemistry; 2. Practical anatomy; 3. Bacteriology; 4. Physiological chemistry, including the analysis of urine; 5. Histology; 6. Physiology, and 7. Pathology.

1. Analytical Chemistry.-The medical student should become acquainted with the physical properties, solubilities and general reactions of the salts of silver, lead, mercury, copper, arsenic, antimony, bismuth, iron, zinc, cobalt, nickle, barium, calcium, potassium, sodium, lithium and ammonium, which are employed in medicine. This knowledge cannot be properly acquired from a study of books alone.

The student must see these compounds, must dissolve them, and must ascertain their incompatibles by precipitating the bases and acids with various reagents. To do this properly, experience has shown that a course of twelve weeks is required. If a shorter time is given to this branch the work must be done superficially and the knowledge is but imperfectly acquired.

2. Practical Anatomy.-Each student should first have a thorough drill in osteology. With the bones before him he must study their size and shape, the nature of their articulations, the points of origin and insertion of ligaments, tendons and muscles and the location of foramina. Then he should carefully, slowly, intelligently, under the eye of a demonstrator, dissect every part of the body, and in doing so he must not only study position, size and physiologcal office of each muscle, but of the viscera, the blood vessels and nerves. My observation has led me to believe that too often dissection means nothing more than a study of myology.

If the student is to become a surgeon it is quite as important that he should know what blood vessels and nerves are to be severed in a given operation as to know what muscles he must traverse with his knife. If he becomes a general practitioner he will need to recall the anatomy of the viscera more frequently than that of the muscles. Every medical school should also offer a course in surgical anatomy. Of course the professor of anatomy dissects before the class and gives especial attention to the hernial region and other parts of the body upon which surgical operations are often necessary; but this is not enough. Such knowledge as this is what our legal friends would designate as hearsay. The positive knowledge can be gained only by the student using the knife himself. Knowledge thus gained becomes a part of himself and arms him with a consciousness of his own resources when he is called upon to do the operation for the first time upon the living body.
The osteology should be taught during the first part of the first year. Then the courses in dissection may follow, while that in surgical anatomy is of most benefit when given later in the course and at times when the student is in attendance upon the surgical clinics.

I think that twenty week's time is none too long for the osteology and the dissections, while four weeks more might be given to the operative work.

Bacteriology.-Practical bacteriology is taught as an under-graduate course in only a very few medical schools, but this is not as it should be. The germ theory is now, and has been for some years, much more than a theory, and the causal relation of certain bacteria to certain infectious diseases has been demonstrated with all the certainty of direct scientific experimentation. We all teach that tuberculosis, diphtheria, typhoid fever, etc., are caused by bacteria; that the detection of these bacteria in many cases offers the only early means of positive diagnosis, and yet only a very few colleges afford any demonstrative instruction in this branch. Every sanitarian speaks of the spread of typhoid fever by contaminated drinking-water, and still of the hundreds of medical students graduated in this country within the past three months only a very small number have any conception of the method or procedure necessary to detect the typhoid germ. The surgeon dwells upon the fact that the pyogenic germs give rise to suppuration, and seldom demonstrates these organisms to his students. Great stress is placed upon the necessity of examining the sputum in suspected tuberculosis, and yet hundreds of graduates of the present year would not know how to stain the germ nor would they recognize it were it stained and placed before them. We teach that in many cases the recognition of Loffler bacillus is the only sure means of the postive recognition of diphtheria, and yet how many teachers make their students acquainted with the practical means of recognizing this organism. There is just cause of complaint on this point. Schools which neglect this branch of instruction are not giving their graduates the proper equipment.

A course of bacteriology embraces the methods of the preparation of the various culture media, of the processes of obtaining pure cultures, of the nature of growths on the various media, of the effect of pathogenic germs on animals, and of the detection and identification of the germs in tissue, in sputa, in drinking-water, and elsewhere. Such a course should begin with the study of the non-pathogenic and proceed to that of the pathogenic bacteria. I have tried the teaching of bacteriology in all the classes of a four year's course, and find that I can teach this subject to the students of the first year as satisfactorily as to those of the fourth year. Indeed, there is an advantage in having this instruction given early in the course. The student who has studied the diphtheria bacillus, or that of a tuberculosis, in the laboratory, and knows the manner of their growth, and has watched their effects upon animals, will listen to his clinical lectures upon these diseases with greater interest and more intelligence than the student whose only knowledge of these organisms is confined to that of their names with some imperfectly understood text-book or lecture description of them. Ten or twelve weeks should be given to the course.

4. Physiological Chemisery.-This course should 
embrace a chemical study of the most important secretions of the body. The test for hydrochloric and lactic acids in vomited matters, the recognition of the digestive ferments and the method of the determination of the activity of the same, and an analysis of the urine and bile, should be included. The student should be made perfectly familiar with the constituents of normal urine. In most medical schools the analysis of urine is limited to the detection of sugar, albumen and bile. Students with such training often mistake epithelial scales for casts, and pronounce every reducing substance found in the urine, sugar. I meet with mistakes of this kind frequently, and have known more than one physician to lose the confidence and respect of the patient and his friends by mistaking an epithelial scale for a cast. A physician is often pardoned for overlooking a serious trouble when it exists, but it is a sad mistake to tell some man that he has an incurable form of Bright's disease or diabetes, when nothing of the kind exists. The patient gets over his fright after a while, but he is not likely to forgive the doctor who has made the blunder.

I believe that analysis of urine should form a part of clinical instruction, but this work should be preceded by a scientific study of normal urine and the scientific methods of estimating the most important normal and abnormal constituents of the urine. The course in physiological chemistry will occupy not less than ten or twelve weeks.

5. Histology.-The medical man certainly must be able to recognize the various tissues of the body by their microscopical appearance. $\mathrm{He}$ must be acquainted with the methods of hardening specimens, making sections, mounting and staining the same. This knowledge can not be acquired from books or in the lecture room. Laboratory instruetion in this branch is a necessity. This knowledge can be gained in a course of six weeks.

6. Physiology.-All medical teachers admit the necessity of practical dissection in acquiring anatomical knowledge, while but few schools give practical courses in physiology. Notwithstanding this, it is certainly true that the medical man needs to employ his physiological knowledge quite as often as he does his anatomical learning. When such instruction is properly given we will have much more intelligence displayed in the practice of medicine. One needs to know the anatomy of the heart in order to detect valvular diseases of that organ, but the number of functional diseases of the beart which one is called upon to treat is certainly greater than that of structural diseases of the same organ, and yet the student in most of our schools has no practical instruction in the innervation of the circulating system. How many of us were able from knowledge gained in our undergraduate course to intelligently apply electricity to any part of the body, to mark out anæsthetic areas which would result from injury to or disease of any nerve, to intelligently interpret the reaction obtained in testing the knee reflex, to properly ascertain the degree of sensation in any muscle, to explain the relation between injury to the floor of the fourth ventricle and the glycogenic function of the liver: or in short, did we have any positive physiological knowledge other than a general idea of the processes of digestion, absorption and elimination? Do we not feel the want of this training in the work of every day? Should we not see to it that our students have this instruction? A six weeks' course in laboratory physiology will in my opinion be of great service.

7. Pathology.-The necessity of practical instruction in this branch will be admitted by all. Such a course should embrace both gross and minute pathology. The bungling way in which post-mortem examinations are of ten performed, and the little information which the medical man usually gets from such an examination, afford abundant evidence of the fact that gross pathology is too much neglected in our schools. Without microscopical pathology the determination of the nature of many growths is quiteimpossible. Let us give six weeks to the deadroom and laboratory courses in pathology.

To sum up I would say that the following laboratory courses are essential:

1. Analytical chemistry, 12 weeks.

2. Pracitical and surgical anatomy, 24 weeks.

3. Bacteriology, 10 to 12 weeks.

4. Physiological chemisty, 10 to 12 weeks.

5. Histology, 6 weeks.

6. Physiology, 6 weeks.

7. Pathology, 6 weeks.

This makes a minimum of 74 weeks. The greater part of this work should precede clinical instruction. Besides the above mentioned courses, therapeutics, including electro-theraputics, is now being taught in some schools largely by laboratory methods, and this tendency will grow.

Laboratory methods will soon largely modify clinical teaching. Amphitheatre clinics are giving way to bed-side and section instruction. Laparotomies and other capital operations are now made by the students in some of our schools on dogs. The surgeon as well as the chemist has his laboratory. The student delivers the alcohol baby from the rubber mother before he is permitted to enter the lying-in room. It is no longer necessary to spoil a hatful of human eyes before one becomes a skillful ophthalmologist.

\section{DISCUSSION OF DR. VAUGHAN'S PAPER.}

\section{BY BAYARD HOLMES, B.S., M.D.,}

SECRETARY COLLEGE OF PHYSICIANS AND SURGEONS, CHICAGO, ILL,

Mr. President and Gentlemen of the Association of Medical Colleges:-The paper of Dr. Vaughan, which has interested us so much, suggests great changes in medical education. During the past year my attention has been called to some of the details of laboratory work which must be faced by every one of you. Allow me to very briefly speak of some of the results of my thoughts and studies.

Laboratory rooms must be light and. roomy. Forty square feet of floor is the least amount which will accommodate a single student, and then only in the chemical laboratory. In all other laboratories at least sixty square feet of floor space, not including aisles, must be allowed each student. In the chemical laboratory students' desks may be placed twenty feet from the windows; in laboratories in which microscopes are to be used, fourteen feet is a maximum.

The ordinary medical class in the larger institutions ranges from one hundred down to thirty, and therefore the laboratory room should be, if lighted on both sides, not less than fifty by forty feet, and it may be lengthened but not widened. At the institution which I represent, our laboratory rooms were 